\title{
An Investigation of Pull-Out Force of Semi-Buried Lotus Roots after Hydraulic Scouring
}

\author{
Rong Zeng ${ }^{1,2}$, Yitao Lin ${ }^{1,2}$, Zhihua Wan ${ }^{1,2}$, Ming Tu ${ }^{1,2}$, Jun Jiao ${ }^{1,2}$ and Guozhong Zhang ${ }^{1,2, *}$ \\ 1 College of Engineering, Huazhong Agricultural University, Wuhan 430070, China; \\ zengrong@mail.hzau.edu.cn (R.Z.); ouzuxiix1@163.com (Y.L.); wanzhihua_83@webmail.hzau.edu.cn (Z.W.); \\ mingtu@mail.hzau.edu.cn (M.T.); jiaojun@webmail.hzau.edu.cn (J.J.) \\ 2 Key Laboratory of Agricultural Equipment in Mid-Lower Yangtze River, Ministry of Agriculture and Rural \\ Affairs, Wuhan 430070, China \\ * Correspondence: zhanggz@mail.hzau.edu.cn
}

check for updates

Citation: Zeng, R.; Lin, Y.; Wan, Z.; Tu, M.; Jiao, J.; Zhang, G. An Investigation of Pull-Out Force of Semi-Buried Lotus Roots after Hydraulic Scouring. Agriculture 2021, 11, 706. https://doi.org/10.3390/ agriculture11080706

Academic Editor: José Pérez-Alonso

Received: 17 May 2021

Accepted: 26 July 2021

Published: 27 July 2021

Publisher's Note: MDPI stays neutral with regard to jurisdictional claims in published maps and institutional affiliations.

Copyright: (c) 2021 by the authors. Licensee MDPI, Basel, Switzerland. This article is an open access article distributed under the terms and conditions of the Creative Commons Attribution (CC BY) license (https:/ / creativecommons.org/licenses/by/ $4.0 /)$.

\begin{abstract}
Hydraulic scouring is the most effective approach to harvest lotus roots, but its application is largely restricted by the low harvesting rate. After hydraulic scouring, some mature lotus roots are still partially buried in the soil. Therefore, it is highly necessary to develop an appropriate approach to harvest semi-buried lotus roots. In this work, we for the first time studied the interaction between semi-buried lotus roots and soil, as well as analyzing the pull-out process and the factors affecting the pull-out force of semi-buried lotus roots. Firstly, a simple testing platform was designed based on the virtual prototype technology and the tests on pull-out force were conducted on five lotus roots with similar shape but different weights, with the burial depth and pull-out speed as the experimental factors. The results revealed that the maximum pull-out force is not significantly affected by the pull-out speed, whereas it is significantly influenced by the burial depth and the surface area of lotus roots. The maximum pull-out force increased with increasing lotus root surface area and burial depth. In addition, the discrete element method was employed to simulate the pull-out process of lotus root at different pull-out speeds. The simulation results indicated that a higher pull-out speed would result in a greater pull-out force at the same displacement of the lotus root from the soil. Both experimental and simulation results revealed that soil adhesion contributes the most to the pull-out resistance. It was also observed that a slight loosening of semi-buried lotus roots could drastically reduce the pull-out force. These results suggest that some kind of mechanical structure or improvement of water flow can be applied to the existing lotus root harvester to reduce the adhesion between lotus roots and soil. Overall, our findings provide a novel direction for optimizing hydraulic harvesting machines of lotus roots.
\end{abstract}

Keywords: semi-buried lotus root; pull-out force; hydraulic scouring; soil adhesion

\section{Introduction}

Lotus root is one of the largest aquatic vegetables in China. According to statistics, the planting area of lotus root in China in 2014 was about 500,000 to 700,000 hectares [1]. With crispy and refreshing flavor and rich nutrition, lotus roots are popular among Chinese people. Due to the huge economic benefits, the number of lotus root growers and the planting area have been increasing year by year. Lotus roots are mostly planted in low-lying and rotten fields with deep silt [2]. The special planting environment and growth conditions result in great difficulties in mechanized harvesting. As a result, manual harvest is still the main harvesting method. With the rapid decrease in labor resources and increase in labor cost in rural China, the production cost of lotus roots has increased dramatically, which seriously restricts the scale development of the lotus root industry. Hence, some scholars have developed several simple harvesting machines for lotus roots based on the planting characteristics, and the mechanized harvesting of lotus roots has been partly realized. 
Currently, shovel digging and hydraulic scouring are the two most commonly used methods for mechanized harvesting of lotus roots. Due to the fragile texture and slender shape of lotus roots, harvesting with shovel digging causes high damage and loss. The harvesting machine with hydraulic scouring uses a high-pressure water jet to wash the mud around lotus roots. When the lotus roots are free from the mud, they will float to the water's surface so that the operators can easily pick them up. The pressure of the water jet can be adjusted to reduce damage to the lotus roots. Therefore, hydraulic harvesting machines are more widely applied to the harvesting of lotus roots [3-5]. The shape of lotus root is similar to a drum. Sludge attached to the upper surface of lotus root can be washed away by the water jet, whereas the sludge attached to the lower surface is obstructed and cannot be washed away by the water jet. Due to high viscosity of soil, some lotus roots are still partially bound by sludge, and will be in a semi-buried state and thus cannot float up. These lotus roots need to be harvested manually. To develop a reasonable harvesting method, it is essential to analyze the interaction between the semi-buried lotus roots and soil, which may be best reflected by the pull-out force of semi-buried lotus roots. Aiming to improve the working performance of harvesting machines for crops from soil, research has been focused on the pull-out force of crops from soil. For example, Li et al. designed a device to measure the pull-out force of cotton stalk and analyzed the relationship between the pull-out force and cotton root diameter, pull-out direction and pull-out speed [6]. Similarly, Song et al. studied the factors affecting the pull-out resistance of cotton stalk and proposed that the diameter of the root of cotton stalk and the soil properties had significant influence on the pull-out resistance [7]. Moreover, Xie et al. simulated the pull-out force of leaf vegetables from soil by EDEM software and determined the optimal pull-out force [8]. Based on the simulation results, they also proposed the optimal working parameters of the harvesting machine. In addition, according to the grip strength, Seongin et al. developed a harvesting robot for lettuce [9].

Understanding the pull-out force of semi-buried lotus roots from soil may help to develop effective methods for the subsequent harvesting of semi-buried lotus roots. At present, there has been no research on the pull-out force of lotus roots. Hence, in order to explore the factors affecting the pull-out force and analyze the pull-out process of lotus roots, this paper constructed a simple platform to test the pull-out force of semi-buried lotus roots. The pull-out force of different lotus roots was investigated at different pull-out speeds and burial depths, and the effect of these factors on the pull-out force was evaluated. In addition, the pull-out process of a testing lotus root was simulated by the discrete element method via the EDEM software. Experimental and simulation analysis results suggest the importance of finding an effective approach to reduce the pull-out resistance, so as to improve the harvesting machine with hydraulic scouring for lotus roots.

\section{Materials and Methods}

\subsection{Analysis of Pull-Out Force of Semi-Buried Lotus Roots after Hydraulic Scouring}

After washing by water jet, the lotus roots may naturally float out of the water surface. Taking one lotus root as an example, the floating process is demonstrated in Figure 1. Firstly, the lotus root is buried in a certain depth under the mud (a). Then, the hydraulic harvesting machine starts to wash the mud layer (b), and the mud surrounding the lotus root is washed away (c). Finally, the lotus root is separated from the mud layer and floats out of the water surface (d). Since the burial depth of each lotus root in mud layer is not consistent, the distance between the nozzle of the water jet and each lotus root is uncertain, resulting in the failure of deeper lotus roots to float out. In addition, the water jet of hydraulic scouring will generate a downward impact force. If the mud layer is soft, the impact force may press the lotus roots into a deeper layer, preventing the lotus roots from floating. These lotus roots will be semi-buried in the mud and require further measures to be harvested. 


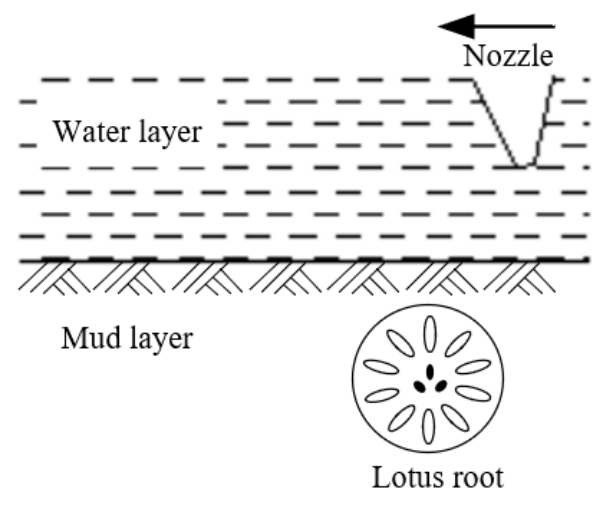

(a)

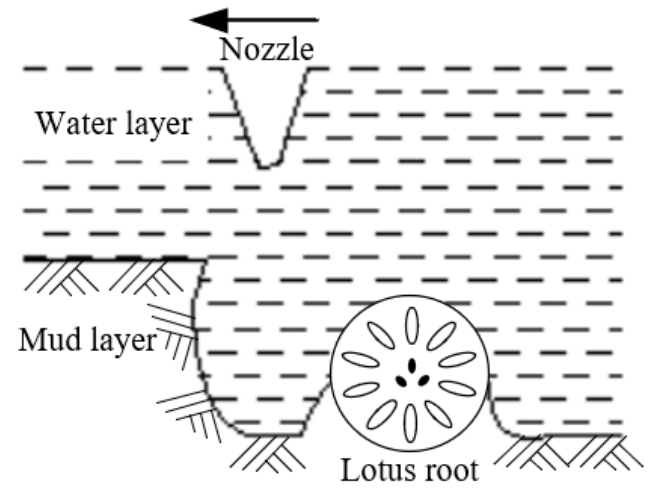

(c)

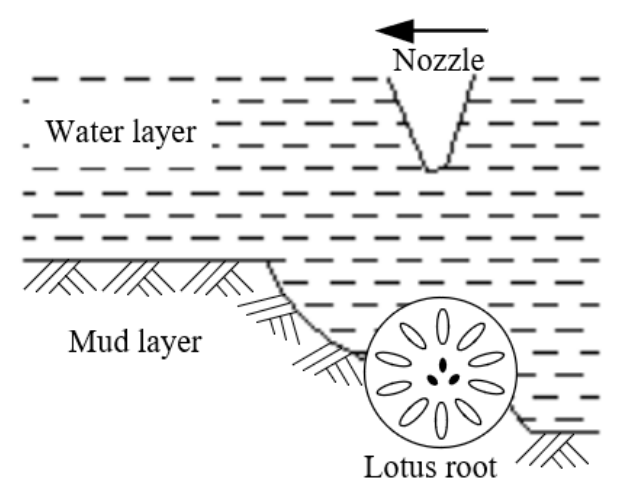

(b)

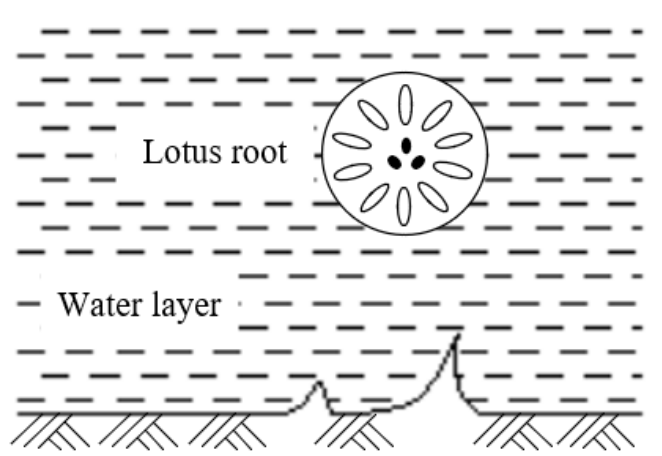

(d)

Figure 1. Floating process of a lotus root. (a) Initial state of the lotus root; (b) Start of floating; (c) Continuous floating; (d) Total floating.

In this section, the forces acting on the semi-buried lotus roots are analyzed. When the semi-buried lotus root tends to move upward, it is under the buoyancy of water, gravity, the soil adhesion force, the normal force of the mud layer and the pull-out force. Taking the cross-section center of the lotus root as the origin to establish the coordinates, the free-body diagram of the lotus root is depicted in Figure 2.

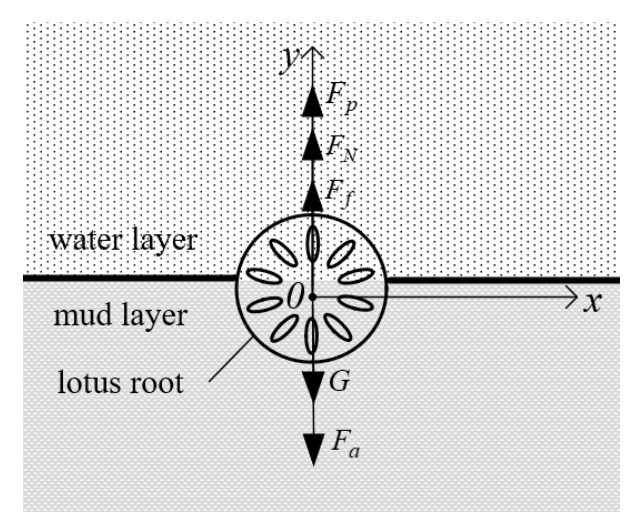

Figure 2. Free-body diagram of the semi-buried lotus root.

Figure 2 shows the vertical force acting on the lotus root when it is pulled out, and the resultant force can be expressed as:

$$
\sum \vec{F}=\vec{F}_{f}+\vec{F}_{p}+\vec{F}_{N}+\vec{G}+\vec{F}_{a}
$$


where $\sum F$ represents the resultant force; $F_{f}$ and $F_{p}$ denote the buoyancy of water and pull-out force, respectively; $F_{N}$ is the normal force of the mud layer; $G$ is the gravitational force; and $F_{a}$ describes the equivalent soil adhesion force in the vertical direction. When the pull-out force is lower than the vertical resistance, the lotus root will stay static in the mud layer, and thus, $\sum F$ is 0 . Therefore, the equilibrium equation is given by:

$$
0=\vec{F}_{p}+\vec{F}_{p z}
$$

and the vertical resistance is:

$$
\vec{F}_{p z}=\vec{F}_{f}+\vec{F}_{N}+\vec{F}_{a}+\vec{G},
$$

where $F_{p z}$ is the pull-out resistance. If the pull-out resistance is overcome, the lotus root will start to float.

\subsection{Pull-Out Force Test \\ 2.2.1. Testing Platform}

A testing platform for the measurement of pull-out force of semi-buried lotus roots is designed in this section. Figure 3 depicts the working principle.

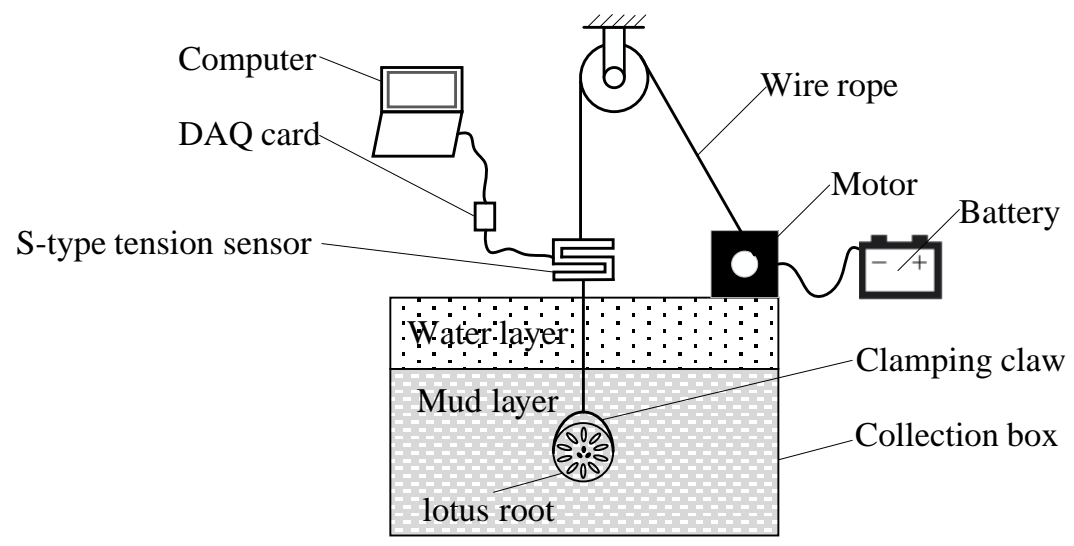

Figure 3. Sketch map of the testing platform.

A single lotus root is buried in the mud layer in the collection box. A clamping claw is employed to grasp the lotus root. The wire rope on the clamping claw is driven upward by the motor to provide a pull-out force. A S-type tension sensor is connected to the wire rope to measure the pull-out force. The output voltage of the tension sensor is acquired by a USB type data acquisition (DAQ) card and converted to pull-out force by a computer with the LabVIEW software. To establish the relationship between the output voltage and the pull-out force, the sensor was calibrated first, and the linear relationship was obtained as:

$$
F_{p}=K U
$$

where $K$ denotes the linear coefficient, which was calibrated to be 872.15 , and $U$ is the output voltage of the S-type tension sensor.

\subsubsection{Experimental Materials}

Lotus roots generally present a drum shape, and there is certain regularity in the diameter distribution. In this experiment, five single lotus roots of the No. 5 Hubei variety were selected as the test objects according to the diameter distribution. The testing lotus roots showed similar shapes but different sizes without obvious damage in appearance. The average length of the five single lotus roots was $22.58 \mathrm{~cm}$, and the minimum and 
maximum diameters were $55.1 \mathrm{~mm}$ and $94.5 \mathrm{~mm}$, respectively. First, their weights were measured and the values are presented in Table 1.

Table 1. Weights of the testing lotus roots.

\begin{tabular}{lccccc}
\hline Lotus Root & No.1 & No.2 & No.3 & No.4 & No.5 \\
\hline Weight $/(\mathrm{g})$ & 789 & 855 & 734 & 696 & 657 \\
\hline
\end{tabular}

The mud layer in the collection box was set as $30 \mathrm{~cm}$ in depth, and the water layer was set as $10 \mathrm{~cm}$ in depth. Soil samples were collected from a lotus root field located at Binhu fishing ground in Dongsheng Town, Shishou City, Hubei Province, China. The soil hardness and moisture content were measured and presented in Tables 2 and 3, respectively. Since the distribution of soil layer was not uniform, the soil hardness was measured five times at different positions to obtain the mean value (Table 2). Table 3 shows the moisture content in different layers of soil. As the surface layer is mainly solid with silt, it has higher fluidity and moisture content. With increasing soil depth, the soil hardness increases, accompanied by a decrease in moisture content.

Table 2. Soil hardness.

\begin{tabular}{ccccccc}
\hline Number of Test & $\mathbf{1}$ & $\mathbf{2}$ & $\mathbf{3}$ & $\mathbf{4}$ & $\mathbf{5}$ & Mean Value \\
\hline Soil hardness $/(\mathrm{kPa})$ & 330 & 350 & 370 & 300 & 330 & 336 \\
\hline
\end{tabular}

Table 3. Soil moisture content (\%).

\begin{tabular}{ccc}
\hline \multirow{2}{*}{ Number of Test } & \multicolumn{2}{c}{ Soil Depth } \\
\cline { 2 - 3 } & $\mathbf{0 ~ 1 0 ~} \mathbf{~ m ~}$ & Beneath $\mathbf{2 0} \mathbf{~ c m ~}$ \\
\hline 1 & 40.25 & 20.82 \\
2 & 48.25 & 22.05 \\
3 & 43.11 & 23.07 \\
Mean value & 43.87 & 21.98 \\
\hline
\end{tabular}

\subsubsection{Experimental Methods}

Since lotus roots are usually buried in a disordered state, different burial depths of lotus roots will require different pull-out force. Therefore, the burial depth was taken as one experimental factor. During the test, the burial depth was defined as shown in Figure 4. Before the test, the testing lotus root was slowly pressed into the mud layer, and the distance between the upmost point of the lotus root and the water layer surface was measured by a tape measure, which was recorded as the burial depth. Then, the mud attached to the upper surface was removed to form a semi-buried state. As the soil hardness increases with increasing soil depth, it is difficult to bury lotus roots to depths greater than $20 \mathrm{~cm}$. Therefore, the burial depths of $15 \mathrm{~cm}$ and $20 \mathrm{~cm}$ were chosen to measure the pull-out force of the semi-buried lotus roots.

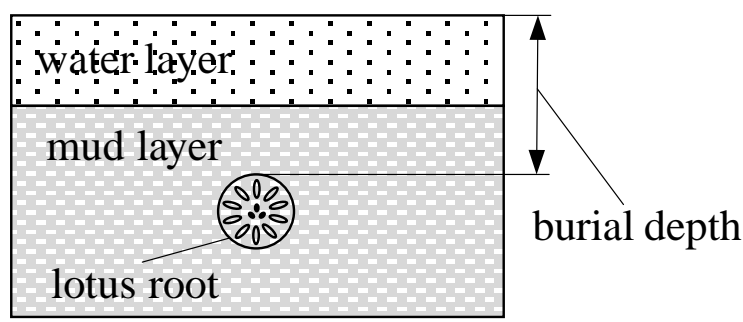

Figure 4. Definition of the burial depth. 
Pull-out speed was another experimental factor to be considered in the test. The adjustment of the pull-out speed was fulfilled by the governor of the motor. The rotational speed of the motor was controlled at $10 \mathrm{r} / \mathrm{min}, 6.6 \mathrm{r} / \mathrm{min}$ and $3.3 \mathrm{r} / \mathrm{min}$, which correspond to the pull-out speeds of $20.9 \mathrm{~mm} / \mathrm{s}, 13.8 \mathrm{~mm} / \mathrm{s}$ and $6.9 \mathrm{~mm} / \mathrm{s}$, respectively. Finally, by taking the buried depth, pull-out speed and different lotus roots as the factors, the pull-out force for the testing lotus roots was measured. The experimental factors are listed in Table 4.

Table 4. Factors for the experiment.

\begin{tabular}{cccc}
\hline Level & Pull-Out Speed/(mm/s) & Burial Depth/(cm) & Lotus Root \\
\hline 1 & 6.9 & 15 & No.1 \\
2 & 13.8 & 20 & No.2 \\
3 & 20.9 & & No.3 \\
4 & & & No.4 \\
5 & & & No.5 \\
\hline
\end{tabular}

\subsubsection{Testing Procedure}

The designed platform was employed to test the pull-out force, and the test site is depicted in Figure 5. Before operation of the motor, the sampling rate of the measurement system was set to $10 \mathrm{~Hz}$. During the test, the motor was adjusted to the required speed and the lotus roots were pulled out by the clamping claw, during which the pull-out force was observed. When the pull-out force exceeded the pull-out resistance, that was, the lotus root broke away from the soil, the test was considered to be finished. The next test was carried out after recovery of the mud layer and five minutes of standing. Since the testing lotus root needed to be buried in the soil again in each test, in order to eliminate the influence of operation, each test was repeated three times, and the average pull-out force was recorded.

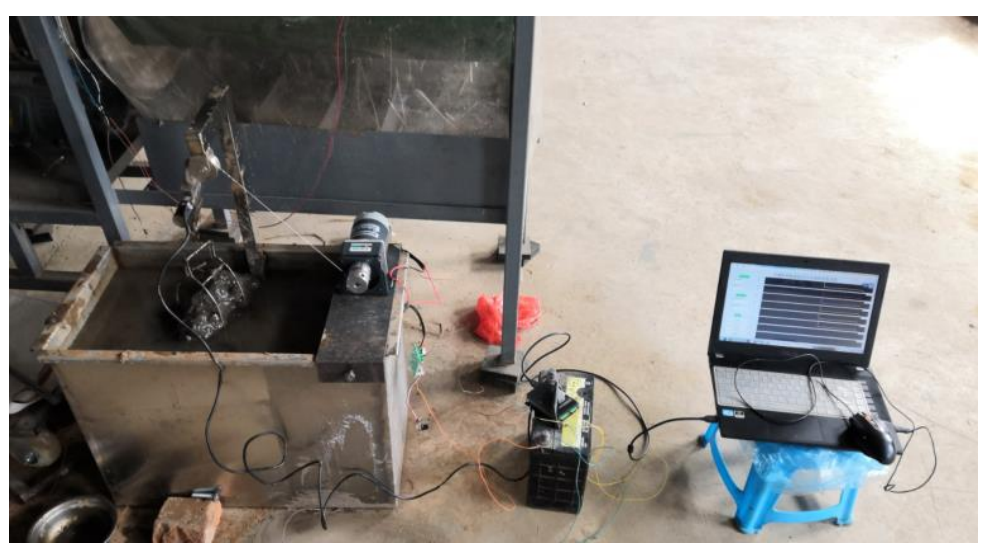

Figure 5. Test site.

\subsection{Simulation of Pull-Out Process of Semi-Buried Lotus Root}

The ultimate aim of the pull-out test is to obtain the value of the pull-out force which can pull lotus root out of the mud layer under a static state. To reveal the changes in pull-out force in the process of lotus root breaking away from the mud layer, the discrete element method (DEM) was used to simulate the pull-out process of semi-buried lotus root, and the simulation was carried out by EDEM 2.6 (DEM Solutions, Edinburgh, UK).

\subsubsection{Lotus Root Model}

A 3D scanner was used to model the lotus root. The surface information of the lotus root was read by the cameras of the 3D scanner and then converted into a '.stl' format file. Then, the SolidWorks 2016 software (Dassault Systemes, Paris, France) was employed to convert the file into a '.stp' file, which can be used in the EDEM 2.6 software. In practice, 
the cameras were set at the top and bottom of the testing lotus root hung by a string. The cameras ran along the length direction of the lotus root to scan the lotus root and generate the 3D model (Figure 6).

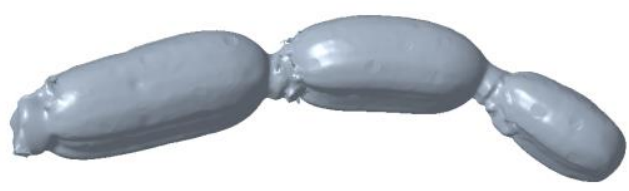

Figure 6. 3D model of the lotus root.

Density is a key parameter that determines the material property of the lotus root in EDEM simulation. Lotus root is not solid, and the inner part is porous and full of air, indicating that pulp density is more applicable to the simulation than bulk density. The pulp volume was estimated based on the pulp area and stomatal area of the lotus root, which can be used to determine the pulp density.

First, the total volume of the lotus root was measured by the drainage method. In order to measure the pulp area and stomatal area, the testing lotus root was cut into several slices along its axial direction, which were then placed on several black papers with a size of $10 \times 10 \mathrm{~cm}$. A camera was then used to capture the images of the slices. Each image was treated and shown in Figure 7. After that, an image with white pulp and another image with white stomata were obtained. From Figure $7 \mathrm{a}$, the area of the pulp part could be determined. In addition, the area of the stomatal part could be measured from Figure $7 \mathrm{~b}$. Accordingly, the ratio of pulp part area to the total area of the section (the area of pulp + stomata) was calculated to be 0.817 . Finally, the pulp volume and the pulp density of the five testing lotus roots could be obtained (Table 5).

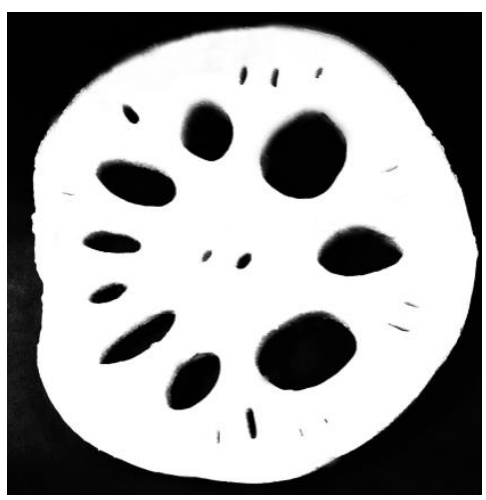

(a)

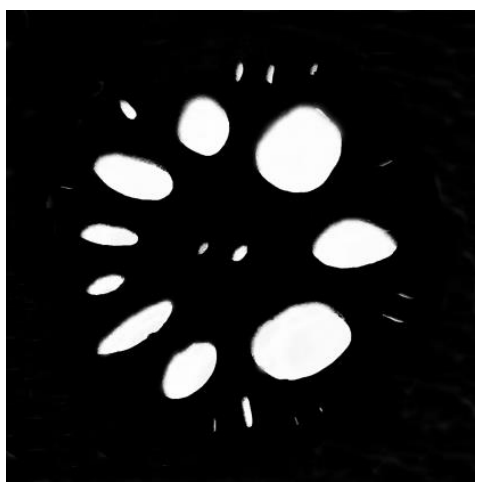

(b)

Figure 7. Images of a slice of the testing lotus root. (a) White pulp; (b) White stomata.

Table 5. Volume and density of pulp.

\begin{tabular}{cccccc}
\hline Lotus Root & No.1 & No.2 & No.3 & No.4 & No.5 \\
\hline Total Volume $/\left(\mathrm{mm}^{3}\right)$ & 919 & 1089 & 877 & 853 & 849 \\
Volume of pulp $/\left(\mathrm{mm}^{3}\right)$ & 751 & 890 & 717 & 697 & 694 \\
Density of pulp $/\left(\mathrm{kg} / \mathrm{m}^{3}\right)$ & 1050.60 & 960.67 & 1023.71 & 998.57 & 946.69 \\
\hline
\end{tabular}

Finally, the pulp density and bulk density were calculated to be about $996.05 \mathrm{~kg} / \mathrm{m}^{3}$ and $818.78 \mathrm{~kg} / \mathrm{m}^{3}$, respectively. Since the bulk density is lower than the water density, the lotus roots can float out from water after being separated from the mud layer.

Xiao et al. and Guo et al. have measured several mechanical properties of lotus roots and demonstrated their anisotropy $[10,11]$. They also demonstrated that there are only small differences in mechanical properties between the axial and radial directions of lotus 
roots. Therefore, lotus roots can be regarded as isotropic to facilitate the calculation. The elastic modulus of lotus root was measured to be $0.451-2.529 \mathrm{Mpa}[10,11]$. Moreover, due to the low elastic modulus and poor compression performance, the Poisson's ratio of lotus roots was set to be 0.47 by referring to the mechanical properties of common materials. The shear modulus of lotus roots can be calculated by Equation (5):

$$
G=\frac{E}{2(1+v)},
$$

where $G$ is the shear modulus, $E$ is the elastic modulus and $v$ is the Poisson's ratio. Thus, the shear modulus of lotus roots was calculated to be $0.39 \mathrm{Mpa}$.

Currently, there has been no detailed research on the mechanical behavior of the interface between soil and lotus roots; moreover, it is difficult to calibrate the contact parameters. In this study, an approximate method was selected to determine the contact parameters in this simulation. The surface of lotus root is smooth, and its hardness is far greater than that of the sludge attached to the lotus root surface. For this reason, the contact between lotus roots and soil can be similarly regarded as the contact between a farm tool and soil. With reference to the parameters of soil-farm tool contact, we set the static friction coefficient, the dynamic friction coefficient and the recovery coefficient of the soil-lotus root contact as $0.5,0.05$ and 0.6 , respectively [12].

\subsubsection{Soil Model}

The soil of lotus root field is characterized by sticky properties. Thus, the JKR model was selected to model the soil. Due to its ability to characterize the contact surface energy of soil particles, the JKR model has been proved to be suitable for modeling cohesive soil $[13,14]$. The soil parameters of the JKR model are presented in Tables 6 and 7 [15-17].

Table 6. Intrinsic parameters of soil.

\begin{tabular}{ccc}
\hline Poisson's Ratio & Shear Modulus/(MPa) & Density/(kg/m $\left.{ }^{\mathbf{3}}\right)$ \\
\hline 0.4 & 408.3 & 1714 \\
\hline
\end{tabular}

Table 7. Contact parameters of soil particles.

\begin{tabular}{ccc}
\hline Dynamic Friction Coefficient & Static Friction Coefficient & Recovery Coefficient \\
\hline 0.2 & 1.16 & 0.15 \\
\hline
\end{tabular}

To improve the calculation efficiency, the soil particles were generated in a $300 \times 150$ $\times 70 \mathrm{~mm}$ box as depicted in Figure 8 . The soil particles were of a sphere shape and were generated based on a radius of $3 \mathrm{~mm}$ with $0.5-1.5$ fold changes. The surface energy of JKR was $3.5-10.5 \mathrm{~J} / \mathrm{m}^{2}[15-17]$.

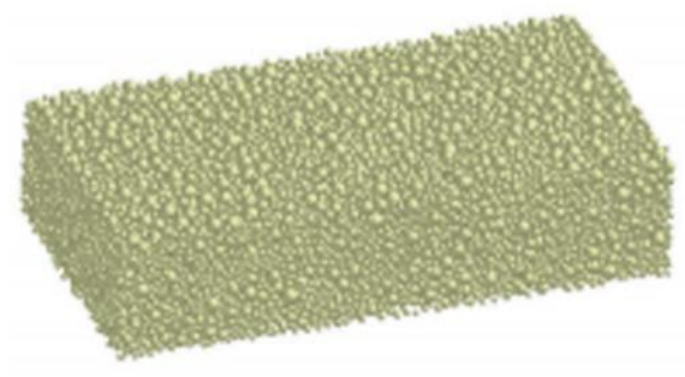

Figure 8. Soil box.

After setting the parameters, a single testing lotus root was semi-buried in the soil model with a burial depth of $15 \mathrm{~cm}$. The EDEM simulation was carried out at the pull-out 
speeds of $10 \mathrm{~mm} / \mathrm{s}$ and $5 \mathrm{~mm} / \mathrm{s}$. In the simulation, the effect of the water layer was neglected. When the lotus root was observed to be completely separated from the soil, the simulation was stopped, and the pull-out force varying with time and displacement was obtained through post processing.

\section{Results and Discussion}

\subsection{Pull-Out Force Testing Results and Analysis}

\subsubsection{Force-Time Curves with Different Testing Factors}

Figure 9 depicts the pull-out force of No.4 lotus root varying with pull-out speed and burial depth. Figure 10 displays the pull-out force of the five testing lotus roots at the burial depth of $20 \mathrm{~cm}$ and the pull-out speed of $6.9 \mathrm{~mm} / \mathrm{s}$.

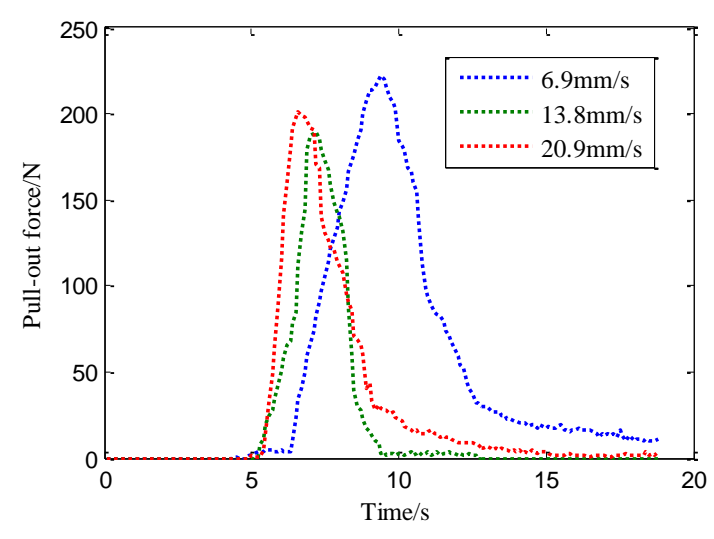

(a)

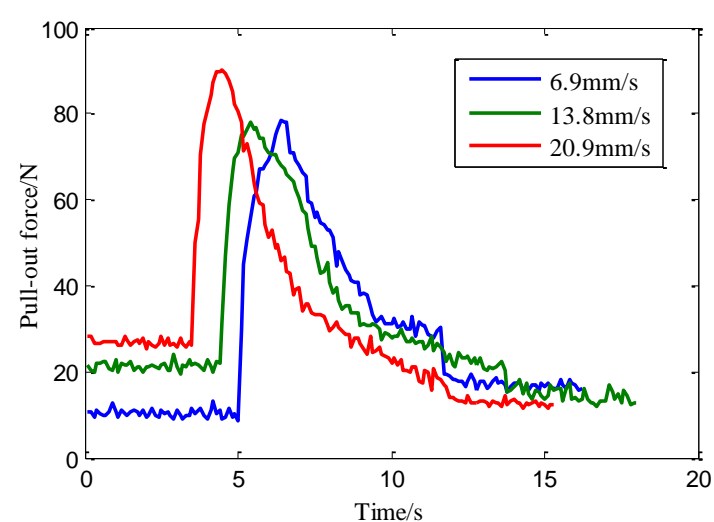

(b)

Figure 9. Force-time curves of No.4 lotus root. (a) Burial depth of $20 \mathrm{~cm}$; (b) Burial depth of $15 \mathrm{~cm}$.

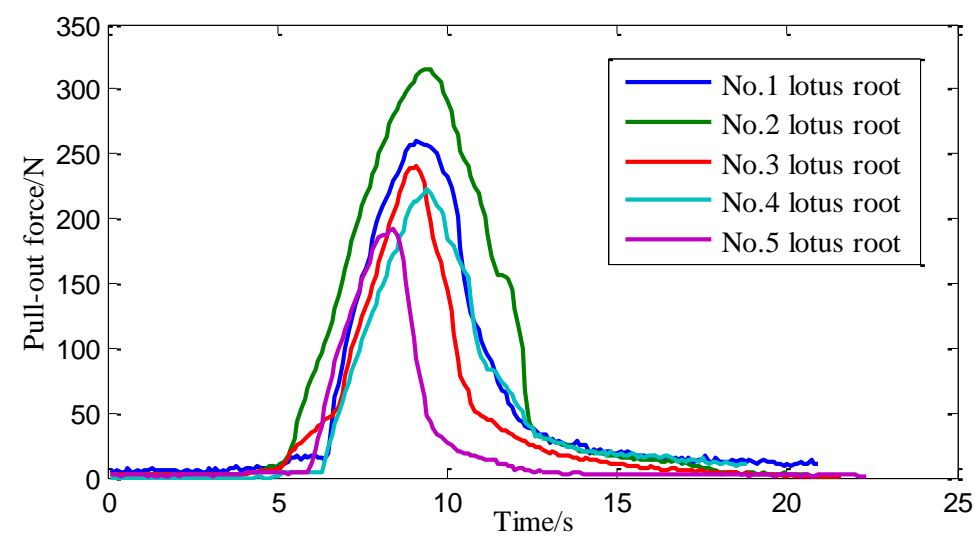

Figure 10. Force-time curves of five testing lotus roots with burial depth of $20 \mathrm{~cm}$ and pull-out speed of $6.9 \mathrm{~mm} / \mathrm{s}$.

Figures 9 and 10 show the pull-out process in the time domain. The pull-out force first increases slowly to reach the peak value with time, followed by a sharp decrease, probably because the lotus root needs to first overcome the resistance, and once the resistance is overcome, the lotus root starts to move and break away from the mud. It can also be discovered that the pull-out force increases with increasing burial depth. Since the moisture content of the mud layer decreases with increasing burial depth, the soil of the deeper layer is harder with a higher viscosity, resulting in a higher resistance due to soil adhesion. In addition, Figure 9 shows that the pulling speed does not affect the maximum pull-out force. Figure 10 indicates that a greater lotus root weight will require a greater pull-out force. 
3.1.2. Average Maximum Pull-Out Force of Each Lotus Root under Different Testing Factors

Figures 11 and 12 present the average maximum pull-out force of each lotus root with different pull-out speeds at the burial depths of $15 \mathrm{~cm}$ and $20 \mathrm{~cm}$, respectively.

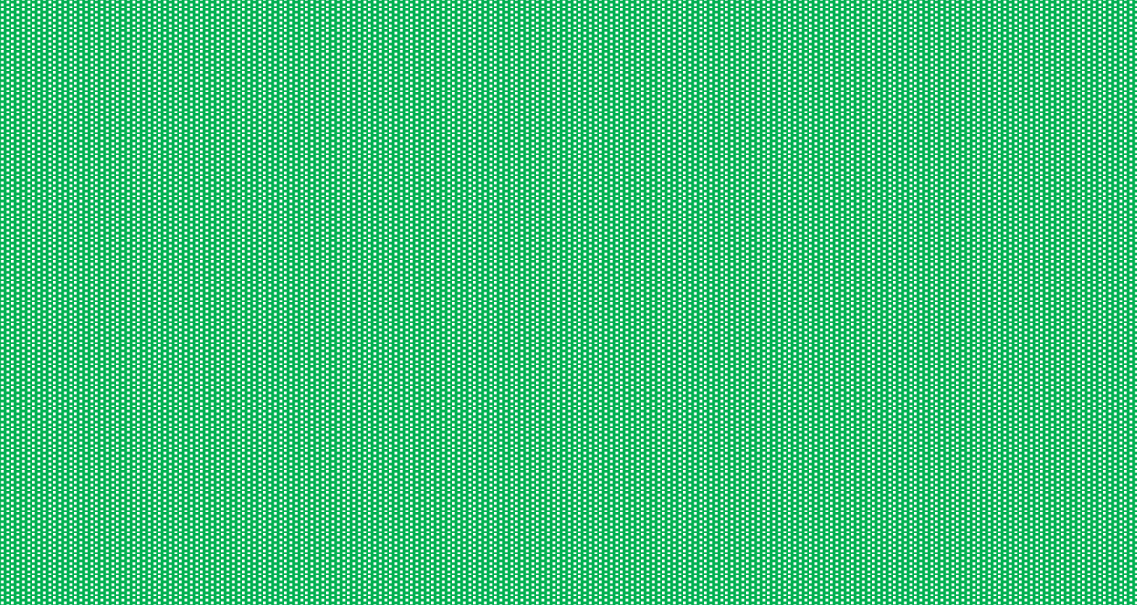

Figure 11. Average maximum pull-out force of different lotus roots at the burial depth of $15 \mathrm{~cm}$.

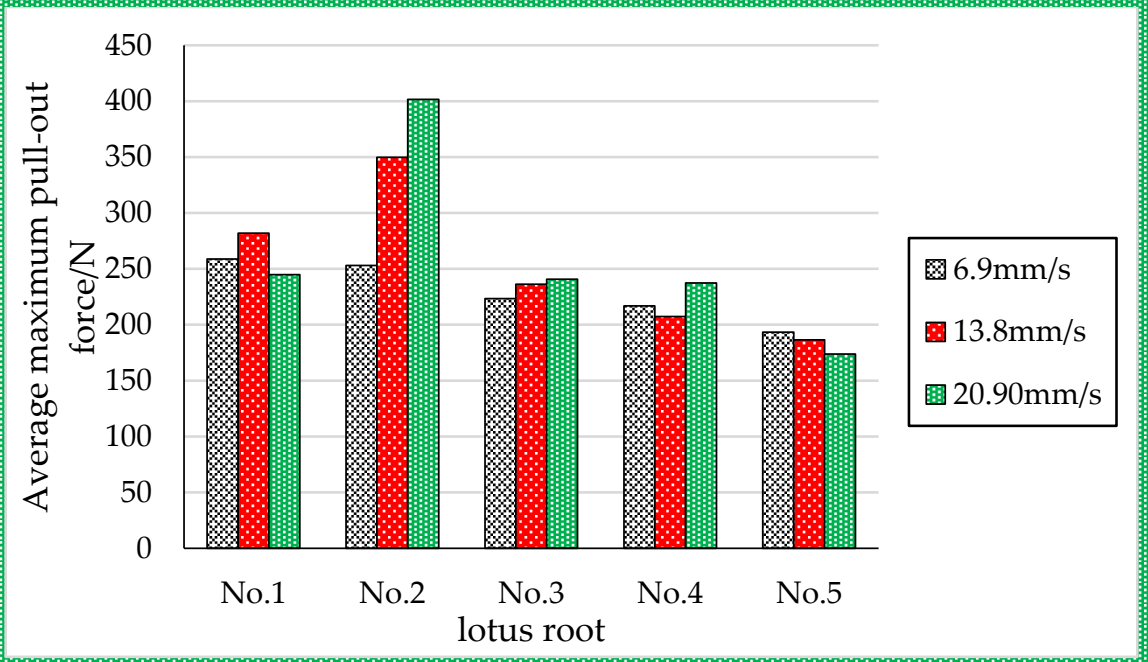

Figure 12. Average maximum pull-out force of different lotus root at the burial depth of $20 \mathrm{~cm}$.

The results show that the average maximum pull-out force varies significantly among different lotus roots. Besides, the burial depth shows significant influence on the average maximum pull-out force, and pull-out speed exhibits no effect.

\subsubsection{Analysis of Variance}

Repeated analysis of variance (ANOVA) was carried out on the average maximum pull-out force of the testing lotus roots under different burial depths and pull-out speeds. The analysis results of No. 1 lotus root are shown in Table 8.

The ANOVA results of other lotus roots are consistent with those in Table 8. According to Table 8, burial depth has a significant impact on the pull-out force. Since the above tests were carried out under a static state, the pull-out speed shows no significant influence on the pull-out force.

Considering the individual differences of the testing lotus roots, the average maximum pull-out force with the burial depth of $15 \mathrm{~cm}$ was analyzed by ANOVA, and the results are presented in Table 9. 
Table 8. ANOVA of the average maximum pull-out force under different burial depths and pullout speeds.

\begin{tabular}{cccccc}
\hline Source of Differences & SS & df & MS & F & Significance \\
\hline Buried depth & $121,054.96$ & 1 & $121,051.96$ & 138.22 & significance \\
Pull-out speed & 993.06 & 2 & 496.53 & 0.57 & \\
Interaction & 1179.08 & 2 & 589.54 & 0.67 & \\
Error & $10,510.03$ & 12 & 875.84 & & \\
Sum & $133,737.14$ & 17 & & & \\
\hline
\end{tabular}

Table 9. AVOVA of the maximum pull-out force of different lotus roots at the burial depth of $15 \mathrm{~cm}$.

\begin{tabular}{cccccc}
\hline Source of Differences & SS & df & MS & F & Significance \\
\hline Different lotus root & $15,697.32$ & 4 & 3924.33 & 22.75 & significance \\
Error & 6898.61 & 40 & 172.47 & & \\
Sum & $22,595.93$ & 44 & & & \\
\hline
\end{tabular}

The results obtained at the burial depth of $20 \mathrm{~cm}$ are consistent with those in Table 9 . The results revealed that different lotus roots have significantly different maximum pullout forces.

3.1.4. Effect of Individual Differences of the Testing Lotus Roots on the Maximum Pull-Out Force

Since individual lotus roots have different surface areas, the contact area between lotus roots and the soil is different, which will result in different soil adhesion force. It is difficult to measure the surface area of each lotus root. Considering the similar shape of lotus roots, the volume of the testing lotus roots can be used to indicate the surface area and the mass. Thus, the relationship between the mass of each lotus root and the maximum pull-out force was analyzed (Figure 13).

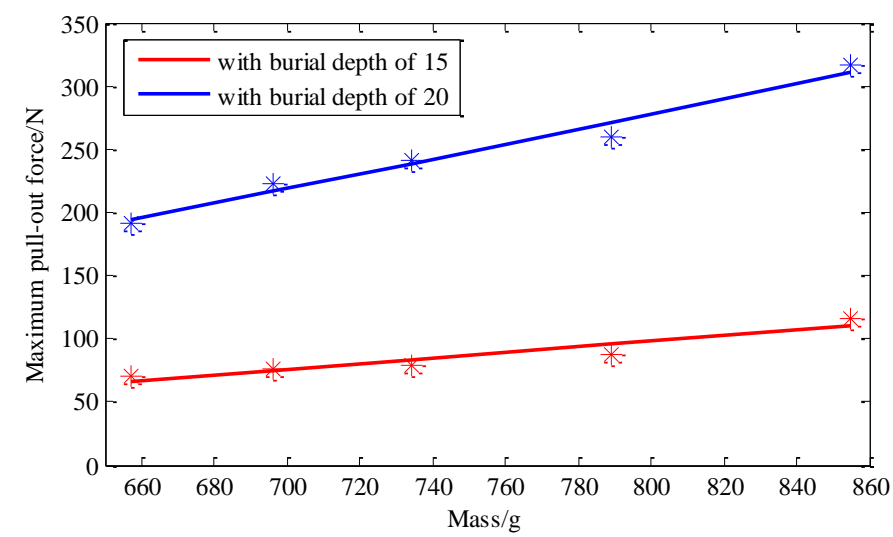

Figure 13. Curve fitting of the maximum pull-out force at different buried depths.

As shown in Figure 13, the maximum pull-out force of each lotus root linearly increases with the mass, indicating that the maximum pull-out force is positively correlated with the surface area of the lotus root. The linear fitting equations illustrating the relationship between the mass and the maximum pull-out force at the burial depth of $15 \mathrm{~cm}$ and $20 \mathrm{~cm}$ can be given by:

$$
\begin{aligned}
& y_{15 \mathrm{~cm}}=0.2236 m-80.72, \\
& y_{20 \mathrm{~cm}}=0.5903 m-194.30
\end{aligned}
$$

where $y$ denotes the maximum pull-out force and $m$ represents the mass of the lotus root. The determination coefficients of the equations are 0.9005 and 0.9770 , respectively. 


\subsubsection{Summary and Discussion}

In summary, when the pull-out force is greater than the resistance, the semi-buried lotus root can break away from the mud layer, and the instant pull-out force reaches the maximum value at the moment of separation. In addition, the maximum pull-out force is not affected by the pull-out speed, but is significantly influenced by the burial depth and surface area of the lotus root: it increases with increasing burial depth and surface area of the lotus root.

During the test, it was also observed that the maximum pull-out force greatly decreased when the testing lotus root was slightly loosened in the mud layer. The maximum pull-out force was detected to be only $20 \mathrm{~N}$ when the lotus root was loosened beforehand and then pulled out from the soil at a burial depth of $15 \mathrm{~cm}$. The water penetrates between the loosened lotus root and the soil attached on the lotus root, disrupting the originally closed environment and weakening the adhesion. Furthermore, further pressing the lotus root causes a large increase in the pull-out force, which is similar to the impact of the water jet. The direct impact of the water jet on the lotus root results in an additional downward pressure, making the lotus root further adhere to the soil, which is not conducive to the floating of lotus roots.

Among the components of pull-out resistance, the buoyancy and normal force play a role in helping lotus root to float up, both of which are related to the gravity of buried lotus roots. Since the gravity only takes a very small proportion in the pull-out resistance, the main factor affecting the pull-out force of semi-buried lotus roots is the adhesion between lotus roots and the soil. In this experiment, significant factors such as burial depth and surface area of lotus roots affect the adhesion of lotus roots to the soil. The results show that once the adhesion between the lotus root and soil is destroyed, the lotus root can be pulled out easily.

Due to the uneven burial depth of lotus roots in the soil, some lotus roots are still difficult to float out of the sludge after being washed by the water jet under a certain pressure during harvesting. Too high jet pressure will cause damage to lotus root skin. It is difficult to balance the floating rate and damage rate. According to the above analysis, some mechanical methods can be used to vibrate and loosen the soil after the first hydraulic scouring, or the water jet can be adjusted to weaken the adhesion between lotus roots and the soil. After that, a second small pressure scouring can be used to improve the floating rate of lotus roots on the premise of reducing the damage.

\subsection{Simulation Results Analysis and Discussion}

The pull-out process is shown in Figure 14. During the pull-out process, the pull-out force varying with time and displacement was recorded (Figure 15).
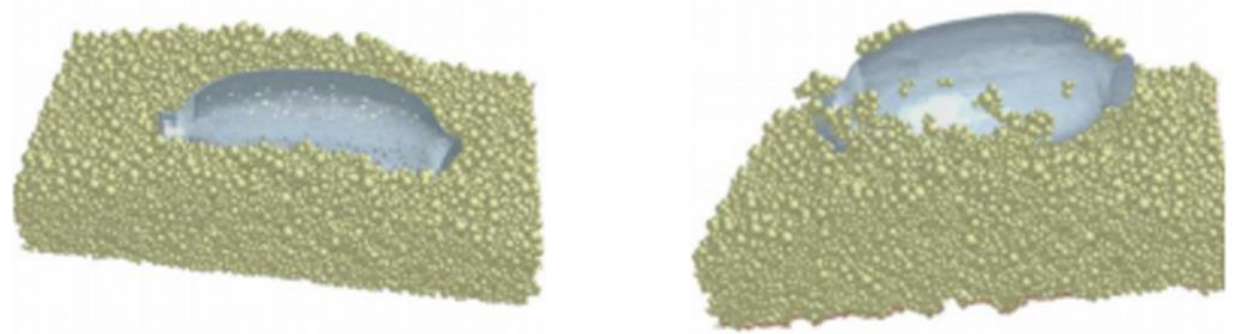

Figure 14. Pull-out process of semi-buried lotus roots in EDEM simulation. 


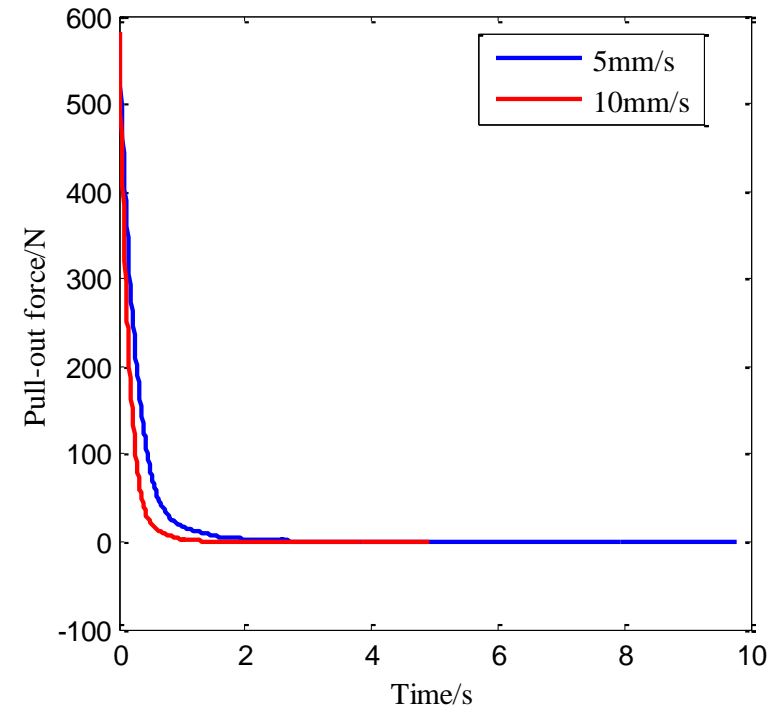

(a)

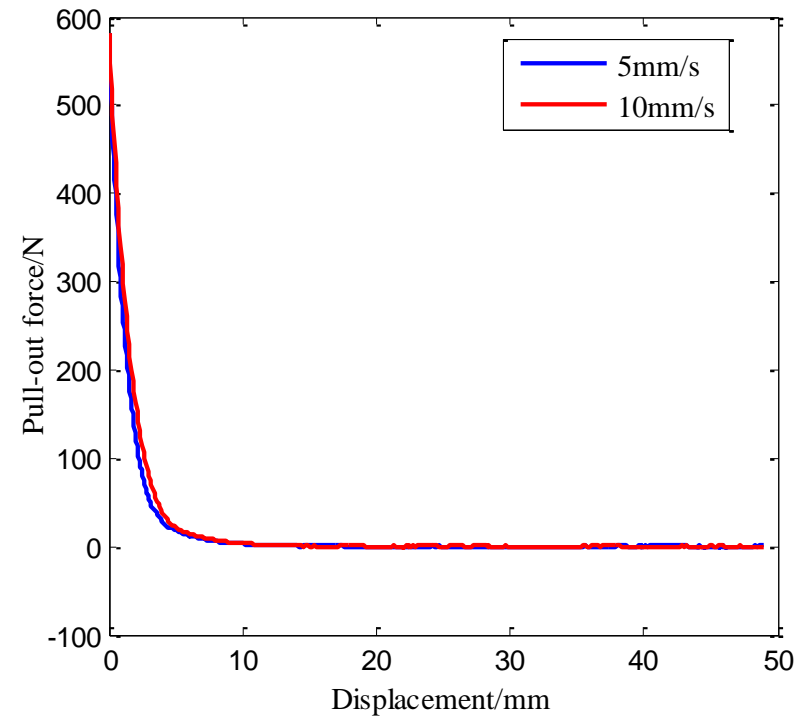

(b)

Figure 15. Pull-out force in simulation. (a) Force-time curve; (b) Force-displacement curve.

Figure 15 shows that the maximum pull-out force of the lotus root in the simulation is greater than that in the test. In the simulation, the water layer is not involved in the soil model, which would result in a larger soil adhesion force. The parameters of soil-soil contact and soil-lotus root contact are the main factors influencing the simulation results. In this study, the soil-lotus root contact parameters were set based on the soil-tool contact in cohesive soil [12]. Furthermore, the soil-soil contact parameters were set based on the properties of the cohesive soil [15-17]. Due to the influence of the water layer, the soil of a lotus root field has certain rheological properties. Hence, the parameters may not accurately simulate the real situation.

The force-displacement curve (Figure 15b) indicates that the pull-out force at the speed of $10 \mathrm{~mm} / \mathrm{s}$ is greater than that at the speed of $5 \mathrm{~mm} / \mathrm{s}$ for the same displacement. For cohesive soil, the adhesion is proportional to the velocity. Similarly, when lotus roots are being moved from the mud layer, a higher velocity usually requires a greater pullout force. When the lotus root begins to break away from the soil, the gap between the lotus root and the soil increases, which will reduce the adhesive force and the pull-out resistance. In this case, the pull-out force decreases rapidly at first, followed by mild decreases until disappearance.

The simulation results also show that the adhesion force of the mud layer mainly contributes to the pull-out resistance. The increase in burial depth and surface area of the lotus root substantially increases the pull-out resistance, resulting in an increase in the maximum pull-out force. Since the adhesion force decays rapidly with the separation of the lotus root from the soil, the required pull-out force will sharply decrease with a slight loosening of the lotus root. The results of simulation are consistent with those of the test.

\subsection{Limitations of the Study}

This research explores the pull-out process of semi-buried lotus roots and analyzes the factors influencing the pull-out force based on experiment and simulation, which can provide a theoretical basis for improving the lotus root harvester with hydraulic scouring. However, there are still several limitations in this study, which need to be further addressed in the future.

In this experiment, the testing lotus root was pulled out in the vertical direction. At this time, the soil adhesion hinders the separation of lotus root and soil, which is also in the vertical direction. If the pull-out direction is adjusted, the direction of soil adhesion will also be changed, and the pull-out effect should be different from that of vertical pull-out. 
However, the test platform proposed in this study can only pull lotus roots out from the vertical direction. Hence, the pull-out angle should be considered in the experimental study to determine the optimal pull-out direction.

Soil samples were collected from a real lotus field in the test and reconstructed according to the actual soil state of the lotus field. However, in the test process, the method of burying the lotus root might cause soil compaction, which may lead to certain deviation of the pull-out force value from that in the actual field. In addition, with the progress of the test, the operation might repeatedly compact and disrupt the soil layer, which may influence the accuracy of the test results.

In the simulation analysis, the parameters for lotus root-soil and soil-soil contact were set by referring to the existing literature, and these contact parameters were not calibrated, which may affect the accuracy of the simulation results.

In the follow-up research, the pull-out force test platform can be improved to make the pull-out angle adjustable and consider hydraulic scouring. In addition, the physical and chemical properties of lotus roots and the mechanical properties of soil in lotus field will be studied to explore the calibration method for the lotus root-soil and soil-soil contact parameters. In the future, we will further explore the interaction among water jet, soil, lotus root, water layer and other relevant factors, so as to provide better solutions for lotus root harvesting with high harvest rate and low damage rate.

\section{Conclusions}

1. A testing platform was designed to observe the pull-out process and acquire the pull-out force for semi-buried lotus roots. The testing platform was proved to be highly efficient and convenient for the real-time measurement of the pull-out force.

2. The testing results show that the pull-out force increases with increasing burial depth and surface area of the lotus root, and the adhesion between lotus roots and soil contributes the most to the pull-out resistance.

3. The discrete element method was employed to simulate the pull-out process of lotus roots from soil. The results also show that the adhesion of soil layer to lotus root is the main factor affecting the pull-out force, and the adhesion decreases rapidly with the separation of lotus roots from the soil.

4. Both experimental and simulation results provide important implications for the improvement of the existing lotus root harvester with hydraulic scouring. To improve the floating rate of lotus roots after hydraulic scouring, proper mechanical structure can be applied after hydraulic scouring, or the water jet can be adjusted.

Author Contributions: Conceptualization, R.Z. and G.Z.; methodology, R.Z. and Y.L.; software, Y.L.; validation, R.Z., Y.L. and J.J.; investigation, R.Z. and Z.W.; resources, R.Z. and M.T.; data curation, G.Z.; writing—original draft preparation, R.Z.; writing-review and editing, R.Z.; visualization, R.Z.; supervision, G.Z.; project administration, R.Z.; funding acquisition, G.Z. All authors have read and agreed to the published version of the manuscript.

Funding: This research was funded by China Agriculture Research System of MOF and MARA, grant number CARS-24-D-02.

Institutional Review Board Statement: Not applicable.

Informed Consent Statement: Not applicable.

Data Availability Statement: The data presented in this study are available on demand from the first author at (zengrong@mail.hzau.edu.cn).

Acknowledgments: G.Z. and the Technical System of National Characteristic Vegetable Industry.

Conflicts of Interest: The authors declare no conflict of interest. 


\section{References}

1. Xie, J.; Han, D.; Wang, J. Development Status Quo and Prospect of China's Lotus Root Industry. Agric. Outlook 2017, 13, 42-45, 51.

2. Liu, M.; Mao, D.; Wang, Z. Research Status of Lotus Root Digging Technology and Prospect of Lotus Root Harvesting Technology in Yellow River Delta Region. Agric. Eng. 2018, 8, 1-5.

3. Wang, C.; Liu, H.; Wang, L. Overall Design of Water Chestnut Harvester. J. Chin. Agric. Mech. 2017, 38, 22-26.

4. Du, J.; Xia, J.; Wu, H. An Investigation of the Performance of Water Jet for Lotus Root Digging Device: Simulation and Experiment. Int. J. Fluid Mach. Syst. 2020, 13, 160-166. [CrossRef]

5. He, J.; Chen, J.; Wang, A. Study on Flow Field of a New Digging Lotus Based on the Vortex Theory. Comput. Simul. 2015, 32, 184-187, 329.

6. Li, Y.; Zhang, G.; Zhou, Y. Design and field experiment of drawing resistance measurement system for cotton stalk. Trans. Chin. Soc. Agric. Eng. (Trans. CSAE) 2013, 29, 43-60.

7. Chen, M.; Song, D.; Wang, Z. Research on the Cotton-Stalk Uprooting Resistance. J. Agric. Mech. Res. $2016,38,64-68$.

8. Gao, G.; Xie, H.; Wang, T. EDEM simulation and experiment of pullout force of protected vegetable harvester. Trans. Chin. Soc. Agric. Eng. (Trans. CSAE) 2017, 33, 24-31.

9. Cho, S.; Chang, S.; Kim, Y. AE-Automation and emerging technologies: Development of a three degrees of freedom robot for harvesting lettuce using machine vision and fuzzy logic control. Biosyst. Eng. 2002, 82, 143-149. [CrossRef]

10. Xiao, K.; Xia, J.; Chen, Z. Biomechanics of lotus root. J. Huazhong Agric. Univ. 2016, 35, 125-130.

11. Guo, Y.; Xia, J.; Xiao, K. Determination and analysis on the yield strength and compressive strength of lotus roots. J. Huazhong Agric. Univ. 2015, 34, 148-151.

12. Yang, N.; An, Z. Simulation and Analysis on Ridging Process of Ridging Machine. Mech. Res. Appl. 2018, 31, 41-43.

13. Gilabert, F.; Roux, J.; Castellanos, A. Computer simulation of model cohesive powders. Influ. Assem. Proced. 2007, 1, 184-194.

14. Johnson, K.; Kendall, K.; Roberts, A. Surface energy and the contact of elastic solids. Proc. R Soc. Lond. 1971, 3, $301-313$.

15. Wu, T.; Huang, W.; Chen, X. Calibration of discrete element model parameters for cohesive soil considering the cohesion between particles. J. South China Agric. Univ. 2017, 38, 93-98.

16. Ucgul, M.; John, M.; Chris, S. 3D DEM tillage simulation: Validation of a hysteretic spring (plastic) contact model for a sweep tool operation in a cohesionless soil. Soil Tillage Res. 2014, 144, 220-227. [CrossRef]

17. Wu, H.; Xia, J.; Zhang, G. Design and experiment of spin-jet flow type lotus root digging machine based on EDEM-Fluent. Trans. Chin. Soc. Agric. Eng. (Trans. CSAE) 2018, 34, 9-14. 